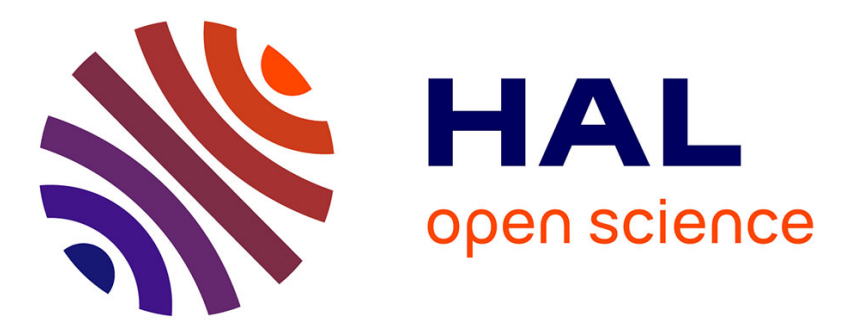

\title{
Boron doped diamond biotechnology: from sensors to neurointerfaces
}

\author{
C. Hebert, Emmanuel Scorsone, A. Bendali, R. Kiran, M. Cottance, Hugues \\ Girard, J. Degardin, E. Dubus, G. Lissorgues, L. Rousseau, et al.
}

\section{- To cite this version:}

C. Hebert, Emmanuel Scorsone, A. Bendali, R. Kiran, M. Cottance, et al.. Boron doped diamond biotechnology: from sensors to neurointerfaces. Faraday Discussions, 2014, 172, pp.47 - 59. 10.1039/c4fd00040d . cea-01828436

\section{HAL Id: cea-01828436 https://hal-cea.archives-ouvertes.fr/cea-01828436}

Submitted on 24 Sep 2019

HAL is a multi-disciplinary open access archive for the deposit and dissemination of scientific research documents, whether they are published or not. The documents may come from teaching and research institutions in France or abroad, or from public or private research centers.
L'archive ouverte pluridisciplinaire HAL, est destinée au dépôt et à la diffusion de documents scientifiques de niveau recherche, publiés ou non, émanant des établissements d'enseignement et de recherche français ou étrangers, des laboratoires publics ou privés. 


\title{
Boron doped diamond biotechnology: from sensors to neurointerfaces
}

\author{
C. Hébert, ${ }^{a}$ E. Scorsone, ${ }^{a}$ A. Bendali, ${ }^{c}$ R. Kiran, ${ }^{\text {ad }}$ M. Cottance, ${ }^{b}$ \\ H. A. Girard, ${ }^{a}$ J. Degardin, ${ }^{c}$ E. Dubus, ${ }^{c}$ G. Lissorgues, ${ }^{b}$ L. Rousseau, ${ }^{b}$ \\ P. Mailley, ${ }^{e}$ S. Picaud ${ }^{c}$ and P. Bergonzo*a
}

Received 14th March 2014, Accepted 14th April 2014

DOI: $10.1039 / c 4 f d 00040 d$

\begin{abstract}
Boron doped nanocrystalline diamond is known as a remarkable material for the fabrication of sensors, taking advantage of its biocompatibility, electrochemical properties, and stability. Sensors can be fabricated to directly probe physiological species from biofluids (e.g. blood or urine), as will be presented. In collaboration with electrophysiologists and biologists, the technology was adapted to enable structured diamond devices such as microelectrode arrays (MEAs), i.e. common electrophysiology tools, to probe neuronal activity distributed over large populations of neurons or embryonic organs. Specific MEAs can also be used to build neural prostheses or implants to compensate function losses due to lesions or degeneration of parts of the central nervous system, such as retinal implants, which exhibit real promise as biocompatible neuroprostheses for in vivo neuronal stimulations. New electrode geometries enable high performance electrodes to surpass more conventional materials for such applications.
\end{abstract}

\section{A Introduction}

Boron doped diamond (BDD) electrodes are extremely promising in the field of biomedical applications as they exhibit a unique combination of properties. Doped diamond synthesis has become a major focus of research and development: undoped diamond exhibits a large band gap and is an electrical insulator at room temperature. When doped with boron, diamond results in an electrode with remarkable electro-analytical properties, which can be used to detect species in solution before oxygen and hydrogen evolution interfere with the analysis. Thus, conductive diamond electrodes take electrochemical detection to new levels, and extend their usefulness to analytes which are not measurable with conventional

${ }^{a}$ CEA, LIST, Diamond Sensors Laboratory, F-91191, Gif-sur-Yvette, France. E-mail: philippe.bergonzo@cea.fr ${ }^{b}$ ESIEE, Université Paris Est, Noisy le Grand, France

'INSERM, U968, Institut de la Vision, Paris, France

${ }^{d}$ MED-EL Research Center, Feodor-Lynen-Srasse 35, 30625, Hannover, Germany

${ }^{e}$ CEA-LETI-DTBS Minatec, 17 rue des Martyrs, 38054, Grenoble 
electrode materials. This opens the field of direct analytical detection with diamond, and in addition to applications where analytes can be detected in biofluids, reverse current spikes can be used to stimulate neural tissues.

There is a real interest in the development of novel neuroprostheses and brainmachine interfaces: these applications witnessed an exponential growth since the success of cochlear implants and of deep brain stimulation for Parkinsonian patients. ${ }^{1}$ Neuroprostheses can either be surface electrodes, meaning the electrodes are simply in contact with the glial surface of the neuronal tissues such as the cortex, or penetrating electrodes, reaching deeper neuronal structures in the brain. Although cortical implants have resulted in a function gain after a few months, this was unfortunately frequently followed by a complete loss of activity. ${ }^{2}$ This loss of functionality could be attributed to a major gliosis occurring around the prostheses or its electrodes in the months following implantation. ${ }^{3}$ Another challenge for neuroprostheses is to increase the resolution of individual electrodes to stimulate more precisely discrete neuronal areas. These objectives motivate the development for new biocompatible materials that limit glial reactions and improve direct interactions with neurones.

Recently, the concept of retinal prostheses was validated in clinical trials, showing that such prostheses can enable blind patients to read short words, identify contrasting objects or follow lanes on the ground., ${ }^{4,5}$ Those retinal prostheses aiming at restoring vision in patients having lost their photoreceptors are either placed in the subretinal space or on the epiretinal side, in direct contact with either the outer or the inner limiting membrane, both produced by glial Muller cells. In the first configuration, the subretinal implant will stimulate retinal bipolar cells (neurones normally postsynaptic to photoreceptors), ${ }^{5}$ whereas epiretinal implants target retinal ganglion cells (spiking neurones that send visual information to the brain via their axon through the optic nerve). ${ }^{4}$ Although these implants have already restored some visual functions in patients, ${ }^{4,5}$ an increase in electrode resolution is required to further improve the restored visual performances, to achieve face recognition, text reading or independent locomotion. Different 3D designs have already been proposed to reach this objective using either pillars ${ }^{6}$ or wells ${ }^{7}$ on the implants.

Among potential new biomaterials, diamond has attracted great attention for its use in neuroprostheses because boron doped diamond exhibits semiconductive properties. Diamond biocompatibility has already been demonstrated with osteoblast cultures ${ }^{8}$ and even embryonic cortical neurons. ${ }^{9,10}$ Embryonic neurons were found to grow selectively on protein patterns stamped on diamond surfaces, ${ }^{9}$ but they could not grow directly on a polished polycrystalline diamond layer or on a nanocrystalline diamond layer unless these layers were coated with peptides or with dispersed nanodiamond particles. ${ }^{10}$ The use of diamond as electrodes has further been demonstrated by recording neuronal activities using cell lines. ${ }^{11}$

\section{B Boron doped diamond electrodes and microelectrode arrays for biosensing}

BDD electrodes possess unique electrochemical properties, namely, a low capacitive background current, a wide potential window in aqueous media, poor adsorption of polar molecules, and corrosion resistance in harsh environments, 
making them highly promising as electrochemical sensors compared to other conventional electrode materials. ${ }^{12-14}$ BDD can be grown using microwave plasma enhanced chemical vapour deposition (CVD) techniques on substrates from 2 to 4 inches using $2.45 \mathrm{GHz}$ plasma excitation. Such layers have demonstrated a bioinertness and long term stability that makes them ideal candidates for biomedical applications..$^{15}$ Due to its carbon nature, BDD also opens the way to the immobilization of functional groups such as DNA, proteins, enzymes etc. on the electrode surface, ${ }^{\mathbf{1 6}}$ thereby bringing high selectivity to the sensing process.

We developed a novel process for the fabrication of diamond microelectrodes that involves the selective growth of diamond over silicon substrates, followed by the deposition of metal contacts and passivation layers. This process includes the initial fixing of detonation diamond nanoparticles over a pre-oxidized 4 inch silicon wafer using a protocol described elsewhere. ${ }^{17}$ Then, an aluminum hard mask consisting of a disc $100 \mu \mathrm{m}$ in diameter was selectively deposited over the areas where the BDD microelectrodes have to be grown. Photolithography enabled us to partially pattern the Al mask, which was used to protect areas of diamond nanoparticles from being etched by a reactive ion etching (RIE) process in oxygen. The $\mathrm{Al}$ hard mask was finally removed and the diamond electrodes were grown. The dimensions of the resulting diamond disc electrodes were typically $300 \mathrm{~nm}$ in thickness and $100 \mu \mathrm{m}$ in diameter. Then, an assembly of Ti $(50 \mathrm{~nm}) / \mathrm{Pt}(150 \mathrm{~nm})$ metal tracks was deposited over the substrate, with a metal ring going around the edge of the diamond disc in order to take an electrical contact from the diamond electrode. Then, a silicon nitride $\left(\mathrm{Si}_{3} \mathrm{~N}_{4}\right)$ passivation layer was deposited by CVD over the substrate. Finally, an opening was made over the diamond electrodes by local etching of the $\mathrm{Si}_{3} \mathrm{~N}_{4}$ layer by RIE with $\mathrm{SF}_{6}$ gas. Typical dimensions range between 10 and $100 \mu \mathrm{m}$ (Fig. 1).

Further to the use of BDD electrodes for the electrochemical detection and quantification of e.g., explosive traces in real media like sea water, ${ }^{18}$ the novel ability to fabricate diamond microelectrode arrays has further enabled the possibility to detect redox active compounds directly in unprocessed media such as urine, wine, milk, blood etc. ${ }^{19}$ e.g. , uric acid (UA) in real urine (Fig. 2 ).$^{20}$ In fact, conventional BDD electrodes are prone to fouling when used in biological (urine, blood plasma) and synthetic fluids. Kiran et al. ${ }^{19}$ have recently proposed an electrochemical treatment where a train of short cathodic and/or anodic pulses are applied to clean fouled electrodes. This technique can be used to retrieve the lost reactivity, characterized by the electron transfer rate $k_{0}$ of the boron doped diamond electrodes, thereby enhancing their reusability over long periods of
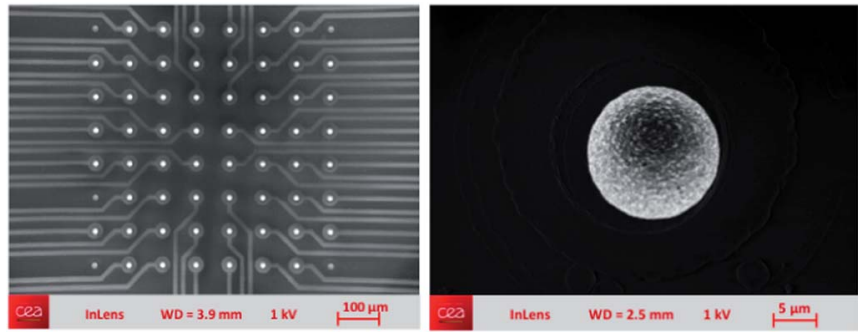

Fig. 1 Image of typical BDD diamond microelectrode arrays. 


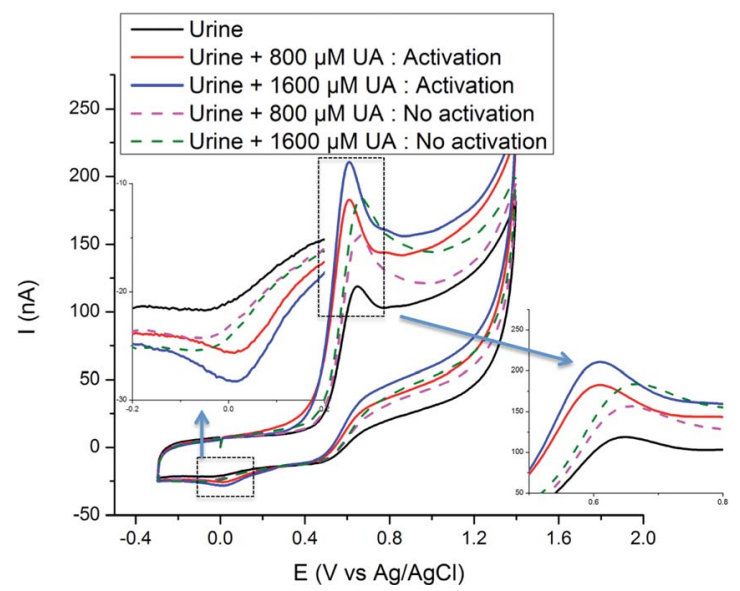

Fig. 2 Cyclic voltammograms of urine diluted 2 fold, and that of diluted urine containing added uric acid $(800-1600 \mu \mathrm{M})$, scanned at $20 \mathrm{~V} \mathrm{~s}^{-1}$. The insets provide details of the oxidation and reduction peaks, displaying the interests of the activation process.

measurements without degradation of the signal, thus significantly extending the field of monitoring and surveying applications. With this approach, uric acid detection in real urine samples was made feasible, with good discrimination of ascorbic acid. Human urine samples were collected from volunteers as real samples for direct BDD uric acid analysis, which was compared to the spectrophotometric method. Measured values using the proposed model were observed to be very close to the spectrophotometric results with a maximum difference of $13 \%$. The interest in continuous monitoring of UA in urine is particularly for patients admitted to intensive care units, where the early diagnostic of acute renal failure can have a major impact on the survival rate of those patients. ${ }^{21}$ In this context, electrochemical detection techniques are seen as a promising alternative to conventional optical methods due to their good sensitivity, fast measuring time, portability, low power consumption and cost effectiveness, thus enabling, for example, direct bedside monitoring.

\section{Boron doped diamond microelectrode arrays for recording of neuronal signals}

Electrophysiology is the study of the electrical properties of cells and tissues, which involves the measurement of the voltage change of a biological entity. It is a powerful approach, not only to study the electrical activity of animal cells to understand the workings of the nervous system, brain, hypothalamus, etc., but also to diagnose and treat nervous system disorders. The electrical activity of neurons can be measured directly using extracellular microelectrode arrays. The potential changes in the vicinity of the electrode, caused by currents flowing across the neuronal membranes of multiple neurons, can be detected. The same microelectrode arrays as presented in the previous section can be used in order to record the invoked activity of cell signals. For example, arrays of 64 pixel diamond microelectrodes have successfully been used by Maybeck et al. ${ }^{22}$ for recording of 
the cardiomyocyte-like cell line HL-1. The study demonstrated that boron doped nanocrystalline diamond (BNCD) electrodes were able to detect cardiac cell action potentials as well as gold electrodes, while electrodes performed up to a factor of four times better than planar metal electrodes of the same diameter. BNCD electrodes survived the mechanical stresses of contractile cells without the defects commonly observed in other nanostructured materials after cell culture (loss of platinum black, removal of CNTs, ${ }^{23}$ and tipping of pillars ${ }^{24}$ ). This enabled us to conclude that the diamond layer remained on the surface during cell contraction by the lack of thinning-induced defects after multiple cultures and the contractile tension generated in mature HL-1 cells (the cells did not pull themselves off of the surface), thus demonstrating that the diamond MEA's physical robustness makes it a durable and easy to handle device for repeated or long-term use, with promise for recording electrical signals from biological samples in addition to its established role in electrochemistry.

\section{Boron doped diamond microelectrode arrays for neurostimulation and implants}

The main constraint where in vivo tissue stimulation is concerned is that the microelectrode array must conform to the size and shape of the tissues to be stimulated. This requires specific developments to enable the fabrication of devices that, further to providing the advantages of previous ones, must also be flexible and exhibit a long connecting foil, enabling the electrical signals to be taken from outside the body to the in vivo neuronal tissues. This is particularly demanding in the case of retina implants since the curvature is particularly high and the surgery constraints impose very strict specifications. In spite of this, the past decade has seen an explosion of research efforts into retinal prostheses aimed at restoring sight to patients blinded by retinitis pigmentosa or age-related macular degeneration. In addition to classical approaches, novel technologies are being explored, often in the context of newly founded commercial enterprises. Also, for the devices to offer a real breakthrough to blind people, theoretical studies have demonstrated that the ability to reach stimulation structures of 600 pixels would be of great benefit as it should be sufficient for patients to read. ${ }^{25}$ This extreme number is not reachable today with current technologies. Several approaches have been proposed, with systems offering typically $60^{26-28}$ to $49^{29-31}$ pixel electrode arrays, with often partial clinical success in short term tests.

In a similar approach as for the fabrication of diamond MEAs used for recording applications, here, nanocrystalline diamond was processed using standard nanotechnology approaches for the fabrication of pixelized microelectrode arrays, but the structure was grown on a sacrificial layer (e.g. $\left.\mathrm{SiO}_{2}\right)$. This process is illustrated through a $3 \mathrm{D}$ approach, in Fig. 3. In brief, after diamond electrodes are deposited on such a sacrificial layer, the standard processing of a soft material such as polyimide is made possible on the substrate. Feed lines to contact each electrode can then be embedded in several layers of soft polyimide, to lead, after removal of the sacrificial layer, to a flexible microelectrode array with high flexibility for retinal stimulation as shown in Fig. 4A. The preparation of such implants has been made feasible with polyimide as well as with parylene. We typically fabricated matrices of 64 independent pixels for the stimulation of 
1- $\mathrm{KOH}$ etch and oxidation

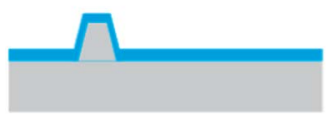

2- Diamond growth

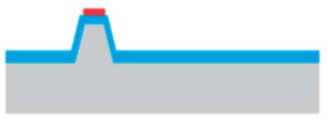

3- Metallic contacts depostion

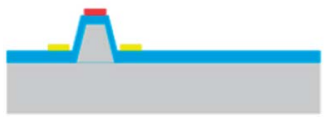

4- Polyimide growth and patterning

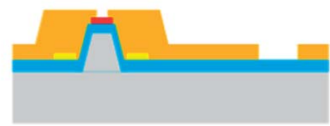

5- Metallic wires and patterning

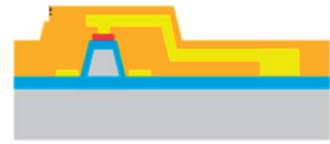

6- Implant lift-off

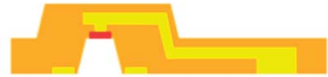

Fig. 3 Process for the fabrication of 3D nanocrystalline diamond implants.

tissues, which were evaluated on laboratory animals in the form of preliminary prototypes of diamond implantable electrode devices (Fig. 4).

\section{E Non-planar diamond electrode matrices towards the improvement of spatial resolution}

Furthermore, a study driven by the Vision Institute in Paris rendered the specifications more challenging, as Djilas et $a .^{7}$ demonstrated the benefit of fabricating 3D wells where a small group of retinal cells could be stimulated, in order to improve the stimulation precision. In fact, the major challenge in visual

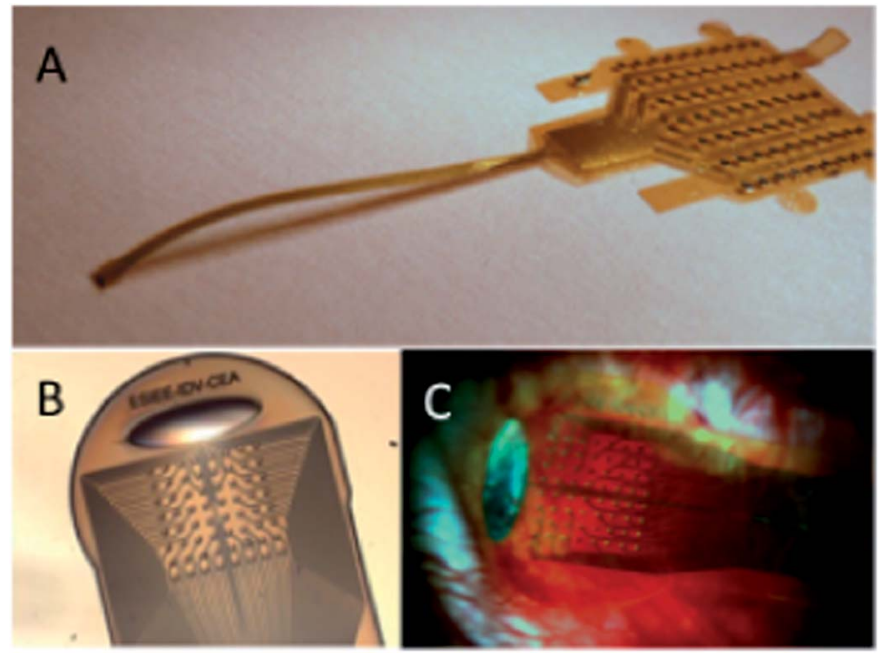

Fig. 4 (A) A view of a non-3D diamond MEA implant for retinal stimulation. The left part consists of 64 diamond electrodes as shown in (B). (C) An in vivo MEA array as implanted in $\mathrm{P} 23 \mathrm{H}$ rats at the Paris Vision Institute. 
rehabilitation using neuroprostheses is to improve the spatial resolution of the stimulated area of each individual electrode, which is a direct consequence of the density of independent pixels. It has been demonstrated that the electrode resolution can be improved using bipolar stimulations between two neighbouring electrodes rather than with a configuration based on a stimulating electrode and a distant common ground..$^{32}$ Using a retinal implant where each pixel exhibits a 3D geometry, one could further improve such bipolar stimulations by locally maintaining neurons between both stimulating electrodes ${ }^{33}$. The success of such 3D designs implies that the residual blind retina remains sufficiently plastic to shape itself around the electrodes. This plasticity of the blind residual retina was demonstrated using pillars penetrating the tissue or cavities filled with cells. ${ }^{34}$ However, the neuronal nature of the cells surrounding pillars or located in the cavities was not fully assessed, since cell immunolabelling could not be performed within this approach. In addition, bipolar stimulation would require two wires per pixel, thus significantly increasing the complexity of the implant design when the number of pixels is increased. An alternative approach proposed the use of a returning ground grid surrounding each of the individual stimulating electrodes, thus enabling improved localisation of the stimulating current providing the grid material exhibits a low impedance. ${ }^{34}$ Electrical stimulations have to be constrained to small tissue areas, as proposed in three-dimensional arrays aimed at confining the current within a small volume around the stimulating electrode. ${ }^{6}$ A previous study by our group validated in vivo a model of 3D well shaped electrodes, demonstrating the favourable integration of the retina cells within such cavities when designed at optimized sizes. ${ }^{7}$ This modelling was based on the hypothesis that the neuronal tissue would shape itself into the $3 \mathrm{D}$ wells located within the implant.

To address these challenges, Rousseau et al. ${ }^{35}$ have proposed novel approaches enabling the synthesis of 3D shaped nanodiamond microelectrode arrays. The goal is to achieve an implant with each electrode placed at the bottom of a typically $30 \mu \mathrm{m}$ deep recess and a ground located at the surface of the implant. To fabricate this 3D structure, we have chosen to use silicon moulds fabricated by $\mathrm{KOH}$ etching of silicon $<100>$ substrates. A specific design with compensation structures was added to obtain these moulds. Initially a simple structure without electrodes was made for histological testing as well as to check the plasticity of the retina. A silicon wafer was oxidized in a furnace at $1050{ }^{\circ} \mathrm{C}$, in a wet environment with a gaseous mixture of hydrogen and oxygen, for $50 \mathrm{~min}$, in order to get $500 \mathrm{~nm}$ of silicon dioxide. A photolithography step was done to define the patterns for $\mathrm{KOH}$ etching. Silicon dioxide not covered by photoresist was etched away in a solution of buffered HF (10\%). Then, the wafer was placed in $\mathrm{KOH}$ solution and kept at $80{ }^{\circ} \mathrm{C}$ for approximately $30 \mathrm{~min}$ so as to obtain a structure $30 \mu \mathrm{m}$ high. After cleaning, the wafer was oxidized once more under the same conditions to obtain a thick silicon dioxide layer $(1 \mu \mathrm{m})$. Selective growth of diamond could then be initiated, as described above, by spreading a colloidal solution of diamond nanoparticles in deionised water onto the substrate, followed by spin coating. ${ }^{36}$ Nevertheless, due to the topology of the 3D substrate used here, spin coating cannot be used, and an alternative approach based on electrostatic grafting of the diamond nanoparticles was used instead. ${ }^{37}$ This latter method is based on electrostatic interactions between the diamond nanoparticles and a substrate coated with a polyelectrolyte. Thus, the substrates were dipped into poly diallyldimethyl- 
ammonium chloride (PDDAC) polymer aqueous solution. The substrates were then washed with water and dried prior to immersion in the colloidal solution of nanoparticles. Finally, the substrates were washed again with water and dried under $\mathrm{N}_{2}$ flux, resulting in 3D structures completely covered by diamond nanoparticles. A short diamond growth was then performed to fix the diamond nanoparticles on the surface. The patterning of the diamond electrodes was ensured from the direct diamond nanoparticle patterning before growth. ${ }^{38}$ A 500 $\mathrm{nm}$ thick aluminium film was sputtered over the substrate and patterned by photolithography, followed by chlorine plasma etching so as to define the electrode areas. The diamond particles not covered by the aluminium film were etched away by classical RIE in a gas mixture of argon and oxygen with a power of $200 \mathrm{~W}$ for $20 \mathrm{~min}$. Lastly, the aluminium protecting the nanoparticles was removed by wet etching and the wafer was placed in a SEKI 6500 CVD diamond growth reactor to selectively grow the diamond electrodes on a 4-inch substrate. Next, polyimide 2611 was poured on the wafer and spin coated to obtain a $10 \mu \mathrm{m}$ thick layer. The polyimide was cured at $450{ }^{\circ} \mathrm{C}$ under nitrogen for $6 \mathrm{~h}$. Then, a metal mask (aluminium, $500 \mathrm{~nm}$ ) was deposited onto the substrate by sputtering. Photolithography with thick photoresist (AZ 4562) was carried out to define the shape of the implant. The aluminium was then etched away by RIE in chlorine and the polyimide layer was etched by RIE with oxygen plasma. The aluminium was removed and the sacrificial layer (a thick silicon dioxide layer) was etched by HF. The implants were finally rinsed and dried.

A complete surgical procedure was developed to implant, in the sub-retinal position, a complete 3D diamond implant. 3D structures were implanted in $\mathrm{P} 23 \mathrm{H}$ rats in the sub-retinal position for 11 weeks. The retina was then explanted along with the implant, and cell labelling was performed. Cell nuclei were labeled by the DAPI stain (blue), while glial cells and retinal bipolar neurons were immunolabelled by the GFAP antibody (grey) and the Go $\alpha$ and PKC $\alpha$ antibodies (green and red), respectively. In Fig. 5, the $\times 20$ magnification view of the retina/implant wholemount allows visualization of the presence of the nuclei stain in all 25 cavities. Confocal analysis was carried out to check the plasticity of the retina. In Fig. 5, the blue cell nuclei are well localised within the implant cavities,

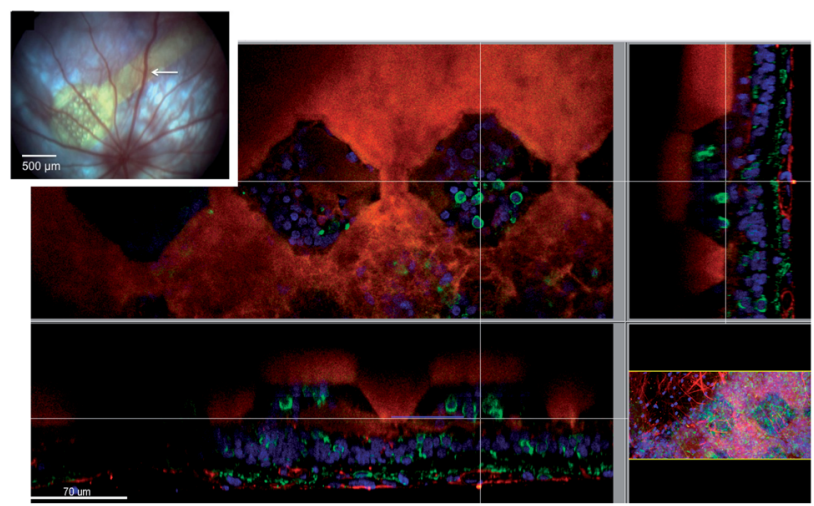

Anti GFAP: Glial Cells ， Anti PKc alpha: Bipolar cells ， Dapi Nuclei

Fig. 5 Result of the histology study on the plasticity of the retina. 
demonstrating that the retina has followed the shape of the implant. Cell labelling revealed that a small group of bipolar cells is present inside the recess.

Further tests were carried out in order to check the biocompatibility of the BDD diamond electrode with the retina. A 3D implant with diamond on the whole surface was fabricated and implanted in a rat subretinal region. As before, cell labeling was performed after several months. The confocal image (Fig. 6) shows that no specific reaction occurs on the tissue and no glial proliferation is visible on the surface. No significant difference is observed here when comparing the sample with our reference (a polyimide implant). Moreover, some bipolar cells can be observed inside each cavity.

\section{F Boosting diamond's electrochemical properties for implants using nanostructured electrodes}

Although diamond exhibits very attractive properties when used as an electrode material, particularly in terms of its electrochemical window, biocompatibility, and resilience to fouling, the material does not match its competitors in terms of impedance and double layer capacitance, which limits the amount of injectable charges. ${ }^{39}$ In fact, values for flat diamond electrodes are generally expected to be around $5 \mu \mathrm{F} \mathrm{cm}^{-2}$, which are extremely low values, comparable to that of flat $\mathrm{Pt}$, although black Pt, which exhibits a 3D texture, can display capacitance values up to $100 \mu \mathrm{F} \mathrm{cm}^{-2}$.

One way to increase the double layer capacitance of the material is to increase its specific surface area. We propose the use of vertically aligned carbon nanotubes with high surface areas as a template onto which boron doped diamond is grown. The resulting composite was found to exhibit a double layer capacitance as high as $0.58 \mathrm{mF} \mathrm{cm}^{-2}$ and very low impedance when compared to planar diamond electrodes in a phosphate buffered saline solution (Fig. 7 and 8). The influence of the CNTs' length as well as the thickness of the diamond coatings were shown to play a very significant role on the electrode performance as discussed by Hebert et $a l .{ }^{40}$ The entire approach relies on the ability to control the seeding and nucleation stage to avoid the etching of the CNT during the first initial stages of growth.

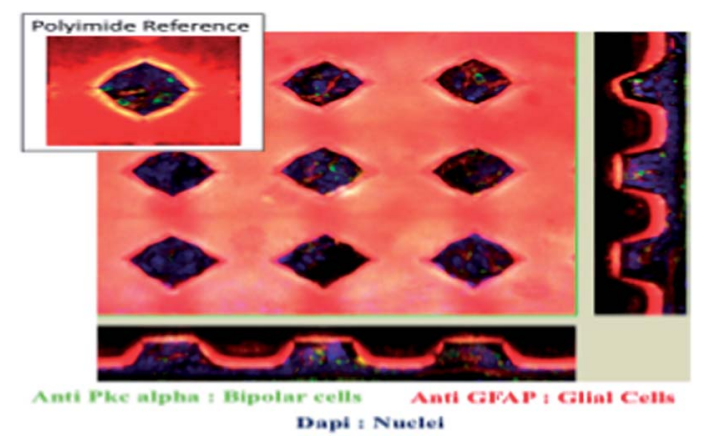

Fig. 6 Biocompatibility test of the BDD material. 


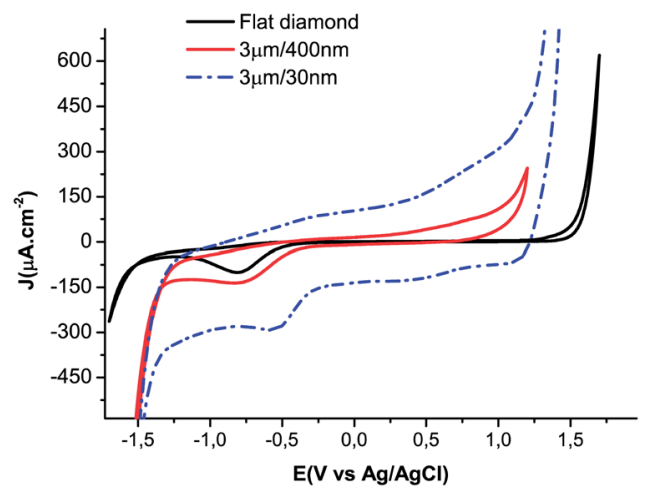

Fig. 7 Cyclic voltammetric $\left(100 \mathrm{mV} \mathrm{s}^{-1}\right)$ electrochemical characterization of BDD coated vertically aligned CNTs in PBS (electrode surface $1 \mathrm{~cm}^{2}$ ).

It is to be noted that such structures exhibit mechanical resilience that is close to that of diamond and far better than CNTs, which are known to be very fragile. As an example, scratching experiments performed with stainless steel tweezers showed that it was possible to not damage the diamond tipped structures. We are currently in the process of implementing such very highly structured electrodes in the shape of microelectrode arrays for neurophysiology evaluation as well as histology experiments.

\section{Conclusions}

From diamond planar electrodes to highly structured surfaces, diamond can take a wide range of forms which are applicable for neurointerfacing and particularly for stimulation purposes. Recent work has been dedicated to the development of retinal interfacing structures for the stimulation of the retina in blind patients. The great progress over the last few years will hopefully enable the validation of interest in diamond and its convincing development. In neuroprostheses, focal stimulation is a real challenge, especially for retinal implants, and we believe this challenge can be successfully overcome by taking advantage of diamond's properties. As presented, we have recently investigated a new approach to obtain focal stimulation for sub-retinal implants, based on the fabrication of a $3 \mathrm{D}$ implant
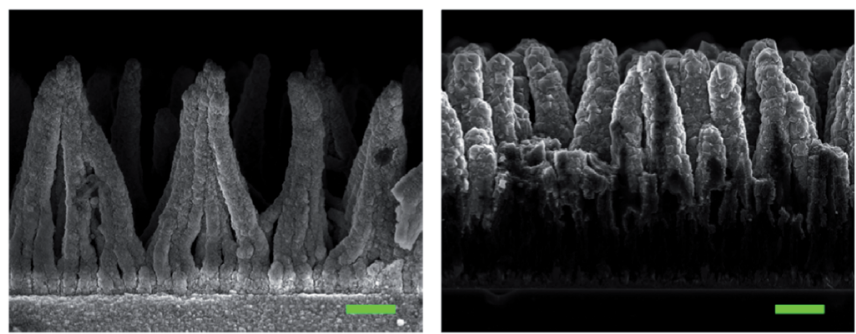

Fig. 8 Cross section of vertically aligned CNT scaffolds covered with diamond (scale bar $1 \mu \mathrm{m})$. 
with electrodes localized at the bottom of a cavity, and a common return ground electrode placed at the surface of the implant. This geometry has previously been modelled to evaluate the efficiency of this $3 \mathrm{D}$ implant. With this structure, the stimulation current is confined within the cavity. Furthermore, we have worked on a new material for long term in vivo stability, namely BDD material. A specific process was optimised to realize a specific implant for histological tests (3D and $3 \mathrm{D}$ with diamond). A complete process was also developed to include BDD electrodes on a soft implant. During in vivo experiments, we have seen that the retina is soft enough to follow the shape of the implant. Using specific cell labelling of the tissues, confocal pictures showed that bipolar cells were present in each cavity of the implant. The tests have so far revealed that BDD material is well accepted by the tissue. A complete surgical procedure for a 3D soft implant was tested to place this implant in the sub-retinal region. The combination of the 3D shape and the BDD electrode's electrochemical performance is expected to lead to significant progress for the next generation of implants. The ultimate challenge for diamond compared to competing materials is the fact that nanocrystalline diamond still exhibits a relatively low capacitance, much lower than that of e.g. black Pt. With the advance of creating a nano-textured material at the hundreds of nanometres scale, recent progress opens up the route for diamond to match the performances of other materials, while it surpasses them with its extreme stability and biocompatibility.

\section{Acknowledgements}

We gratefully acknowledge the funding authorities that have supported this work, namely the Agence Nationale de la Recherche Française via the MEDINAS ANR07TECSAN014 project, as well as the AVIESAN agency for ITS-ITMO IMPLANTS, and more recently the FP7 European commission via the NEUROCARE project no. 280433.

\section{References}

1 M. A. Lebedev and M. A. L. Nicolelis, Brain-machine interfaces: past, present and future, Trends Neurosci., 2006, 29, 536.

2 W. H. Dobelle, M. G. Mladejovsky and J. P. Girvin, Artificial vision for the blind: electrical stimulation of visual cortex offers hope for a functional prosthesis, Science, 1974, 183, 440-444.

3 E. M. Maynard, E. Fernandez and R. A. Normann, A technique to prevent dural adhesions to chronically implanted microelectrode arrays, J. Neurosci. Methods, 2000, 97, 93.

4 M. S. Humayun, J. D. Dorn, A. K. Ahuja, A. Caspi, E. Filley, G. Dagnelie, J. Salzmann, A. Santos, J. Duncan, L. Dacruz, S. Mohand-Said, D. Eliott, M. J. McMahon and R. J. Greenberg, Preliminary 6 month results from the argus II epiretinal prosthesis feasibility study, Conf. Proc. IEEE Eng. Med. Biol. Soc., 2009, 1, 4566-4568.

5 E. Zrenner, K. U. Bartz-Schmidt, H. Benav, D. Besch, A. Bruckmann, V. P. Gabel, F. Gekeler, U. Greppmaier, A. Harscher, S. Kibbel, J. Koch, A. Kusnyerik, T. Peters, K. Stingl, H. Sachs, A. Stett, P. Szurman, B. Wilhelm 
and R. Wilke, Subretinal electronic chips allow blind patients to read letters and combine them to words, Proc. R. Soc. London, Ser. B, 2011, 278, 1489-1497.

6 A. Butterwick, P. Huie, B. W. Jones, R. E. Marc, M. Marmor and D. Palanker, Effect of shape and coating of a subretinal prosthesis on its integration with the retina, Exp. Eye Res., 2009, 88, 22-29.

7 M. Djilas, C. Oles, H. Lorach, A. Bendali, J. Degardin, E. Dubus, G. LissorguesBazin, L. Rousseau, R. Benosman, S. H. Ieng, S. Joucla, B. Yvert, P. Bergonzo, J. Sahel and S. Picaud, Three-dimensional electrode arrays for retinal prostheses: modeling, geometry optimization and experimental validation, $J$. Neural Eng., 2011, 8, 046020.

8 L. B. L. Grausova, A. Kromka, S. Potocky, M. Vanecek, M. Nesladek and V. Lisa, Nanodiamond as promising material for bone tissue engineering, J. Nanosci. Nanotechnol., 2009, 9, 3524-3534.

9 C. G. Specht, O. A. Williams, R. B. Jackman and R. Schoepfer, Ordered growth of neurons on diamond, Biomaterials, 2004, 25, 4073.

10 A. Thalhammer, R. J. Edgington, L. A. Cingolani, R. Schoepfer and R. B. Jackman, The use of nanodiamond monolayer coatings to promote the formation of functional neuronal networks, Biomaterials, 2010, 31, 2097-2104.

11 P. Ariano, A. Lo Giudice, A. Marcantoni, E. Vittone, E. Carbone and D. Lovisolo, A diamond-based biosensor for the recording of neuronal activity, Biosens. Bioelectron., 2009, 24, 2046.

12 T. Yano, D. A. Tryk, K. Hashimoto and A. Fujishima, J. Electrochem. Soc., 1998, 145, 1870-1876.

13 R. Tian and J. Zhi, Electrochem. Commun., 2007, 9, 1120-1126.

14 G. M. Swain, Anal. Chem., 1993, 65, 345-351.

15 M. Panizza and G. Cerisola, Electrochim. Acta, 2005, 51, 191-199.

16 C. Agnès, S. Ruffinatto, E. Delbarre, A. Roget, J.-C. Arnault, F. Omnès and P. Mailley, New one step functionalization of polycrystalline diamond films using amine derivatives , IOP Conf. Ser.: Mater. Sci. Eng., 2010, 16, 012001.

17 E. Scorsone, S. Saada, J. C. Arnault and P. Bergonzo, J. Appl. Phys., 2009, 106, 014908.

18 J. De Sanoit, E. Van Hove, P. Bergonzo and P. Mailley, Electrochemical diamond sensors for TNT detection in water, Electrochim. Acta, 2009, 54, 5688-5693.

19 R. Kiran, E. Scorsone, J. de Sanoit, J.-C. Arnault, P. Mailley and P. Bergonzo, Boron Doped Diamond Electrodes for Direct Measurement in Biological Fluids: An In Situ Regeneration Approach, J. Electrochem. Soc., 2013, 160, H67-H73.

20 R. Kiran, E. Scorsone, P. Mailley and P. Bergonzo, Quasi-Real Time Quantification of Uric Acid in Urine Using Boron Doped Diamond Microelectrode with in Situ Cleaning, Anal. Chem., 2012, 84, 10207-10213.

21 N. Lameire, W. Van Biesen and R. Vanholder, Nephrology, dialysis, transplantation: official publication of the European Dialysis and Transplant Association, European Renal Association, 1999, 14, 2570-2573.

22 V. Maybeck, R. Edgington, A. Bongrain, J. O. Welch, E. Scorsone, P. Bergonzo, R. B. Jackman and A. Offenhäusser, Boron-doped nanocrystalline diamond microelectrode arrays monitor cardiac action potentials, Adv. Healthcare Mater., 2014, 3(2), 283-9. 
23 Y. Hanein, presented at IBN-2, Forschungszentrum Juelich GmbH, Juelich, Germany (6 Apr 2011), unpublished results.

24 D. Brüggemann, unpublished results.

25 J. Sommerhalder, B. Rappaz, R. de Haller, A. P. Fornos, A. B. Safran and M. Pelizzone, Vision Res., 2004, 44, 1693-1706.

26 G. J. Chader, J. Weiland and M. S. Humayun, Artificial vision: needs, functioning, and testing of a retinal electronic prosthesis, Prog. Brain Res., 2009, 175, 317-332.

27 A. Horsager, G. M. Boynton, R. J. Greenberg and I. Fine, Temporal interactions during paired-electrode stimulation in two retinal prosthesis subjects, Invest. Ophthalmol. Visual Sci., 2011, 52, 549-557.

28 R. A. Fernandes, B. Diniz, R. Ribeiro and M. Humayun, Artificial vision through neuronal stimulation, Neurosci. Lett., 2012, 519(12), 122-128.

29 O. Zeitz, M. Keseru, R. Hornig and G. Richard, Artificial sight: recent developments, Klin. Monatsbl. Augenheilkd., 2009, 226, 149-153.

30 Velikay-Parel, D. Ivastinovic, T. Georgi and R. Hornig, Perceptual Threshold And Neuronal Excitability As Long-term Safety Evaluation In Retinal Implants, Acta Ophthalmologica, 2010, 88(s246), 0.

31 M. Keseru, M. Feucht, N. Bornfeld, T. Laube, P. Walter, G. Rossler, M. VelikayParel, R. Hornig and G. Richard, Acute electrical stimulation of the human retina with an epiretinal electrode array, Acta. Ophthalmol., 2012, 90(1), 1-8.

32 S. Joucla and B. Yvert, PLoS One, 2009, 4(3), e4828.

33 D. Palanker, P. Huie, A. Vankov, R. Aramant, M. Seiler, H. Fishman, M. Marmor and M. Blumenkranz, Migration of Retinal Cells through a Perforated Membrane: Implications for a High-Resolution Prosthesis , IOVS, 2004, 45(9), 3266-3270.

34 D. Palanker, A. Vankov, P. Huie and S. Baccus, Design of a high-resolution optoelectronic retinal prosthesis, J. Neural Eng., 2005, 2, S105-20.

35 P. Bergonzo, M. Bonnauron, G. Lissorgues, L. Rousseau, E. Scorsone, Patent IPC: A61N1/05; C30B1/00; C30B29/04, Application number: FR20100054550 20100609, 2011.

36 E. Scorsone, S. Saada, J. C. Arnault and P. Bergonzo, J. Appl. Phys., 2009, 106, 014908.

37 H. A. Girard, S. Perruchas, C. Gesset, C. Chaigneau, M. Vieille, J.-C. Arnault, P. Bergonzo, J. Boilot and T. Gacoin, Electrostatic grafting of diamond nanoparticles: a versatile route to nanocrystalline diamond thin films, ACS Appl. Mater. Interfaces, 2009, 1, 2738-2746.

38 A. Bongrain, E. Scorsone, L. Rousseau, G. Lissorgues and P. Bergonzo, Realisation and characterisation of mass-based diamond micro-transducers working in dynamic mode, Sens. Actuators, B, 2011, 154(2), 142-149.

39 D. J. Garrett, K. Ganesan, A. Stacey, K. Fox, H. Meffin and S. Prawer, Ultrananocrystalline electrodes: optimization towards neural stimulation applications, J. Neural Eng., 2012, 9(1), 016002.

40 C. Hébert, J.-P. Mazellier, E. Scorsone, M. Mermoux and P. Bergonzo, Carbon, 2014, 71, 27-33. 